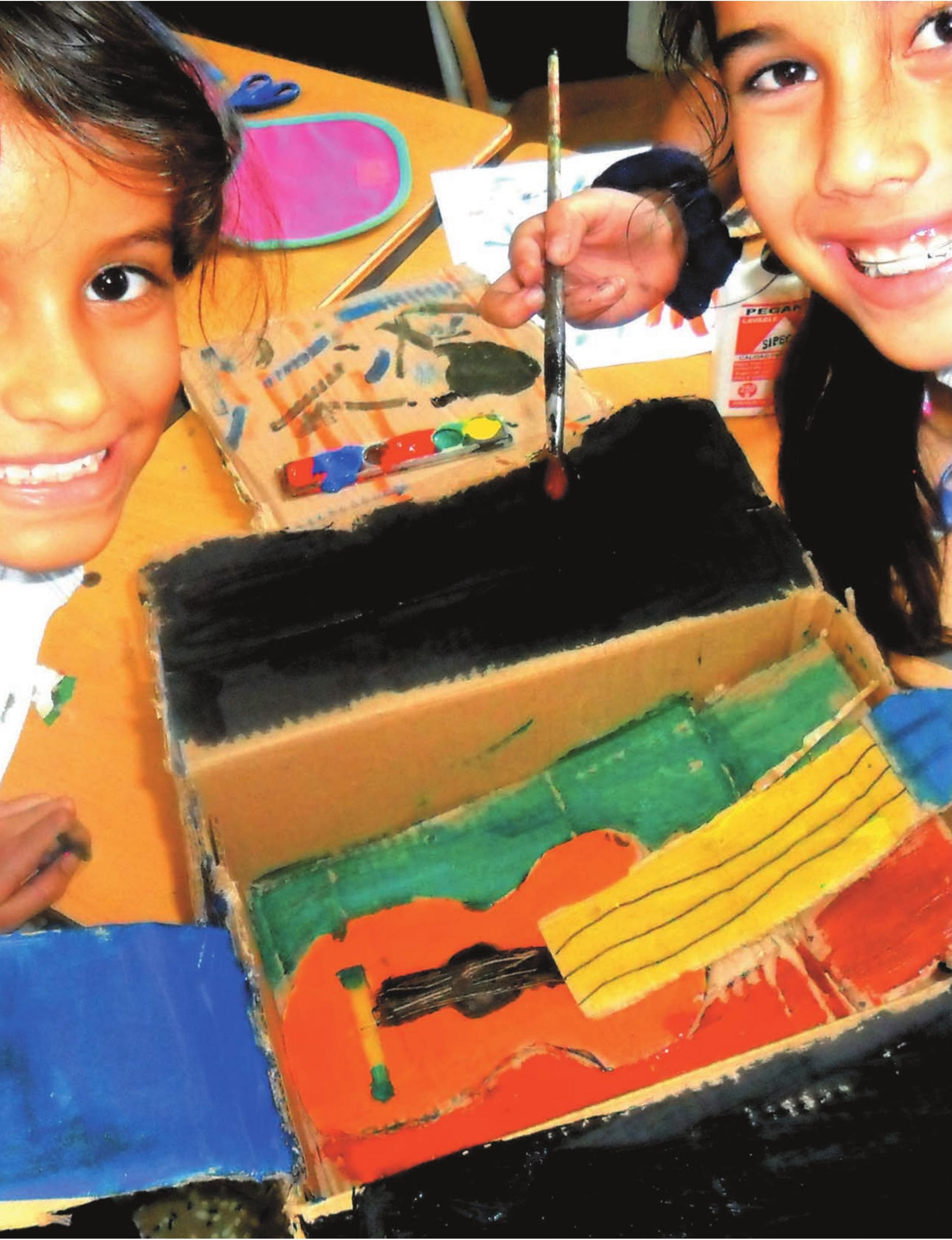





\title{
EL LENGUAJE COMPLEJO DIFERENTE AL COMPLEJO LENGUAJE*
}

\author{
Lucy Garnica Mayorga \\ Psicóloga \\ Especialista en Pedagogía y Semiótica. \\ Docente UPB y Universidad Santo Tomás. \\ Estudiante de la Maestría en Psicología Universidad Pontificia Bolivariana-Bucaramanga. \\ lucy.garnica@gmail.com
}

\begin{abstract}
Resumen
El recorrido que ha tenido el estudio del lenguaje, es un camino de grandes encrucijadas al igual que el estudio del hombre. No en vano en muchas de las referencias que se tienen en estos aportes se involucra gran parte de las ciencias sociales y humanas. ¿Cuál es el indicador común en estos aspectos? ¿Por qué y para qué el interés de tantas áreas del conocimiento en torno al lenguaje? El presente artículo esboza algunas ideas fundamentales para dejar abierta la perspectiva de continuidad en el estudio desde el pensamiento complejo y las actuales teorías del comportamiento humano. Su interés es la construcción de una real perspectiva transdisciplinar como la historia de su estudio lo ha señalado.
\end{abstract}

Palabras clave: Lenguaje complejo, signo, función comunicativa, cognición, lenguaje simbólico

\begin{abstract}
The journey that the study of language has taken is like a road with large intersections the same as the study of mankind. Not in vain many of the references in these contributions have involved most of social and human sciences. What is the common indicator in these aspects? Why and what is the interest of so many areas of knowledge about language? This article outlines some basic ideas to leave open the prospect of continuity in this study from the complex thought and the current theories of human behavior.
\end{abstract}

Keywords: Complex language, sign, communicative function, cognition, symbolic language

His interest is to build a real transdisciplinary perspective as the history of its study has indicated.

"Vaca no es animal, es el pan de cada día que uno sueña masticar"

Piero

\section{Introducción}

La siguiente reflexión en torno al lenguaje, surge de la necesidad de conjugar la búsqueda de sentido latente sobre el tema; pero su dinamismo, inestabilidad, flexibilidad y complejidad no lo permiten por mucho tiempo.

El recorrido que se describe, plantea varios elementos recurrentes y otros que aparecen para volver al mismo lugar de partida. Esa es la magia del lenguaje, sus posibilidades son infinitas y sus incidencias han sido determinantes para el estudio de las manifestaciones del ser, desde que el hombre lo recuerda. Incluso, el lenguaje es el que hace que esto sea posible. Sólo a través de él se obtienen evidencias del pensamiento y sentimientos. Y, sólo a través de él, se ha logrado el avance de la humanidad en ciencia, tecnología, arte (en todas sus dimensiones: plástico y poético), estética, y ética. Como lo expresa Echeverría (1994), los seres humanos son seres lingüísticos; lo social para todos se constituye en lenguaje. $\mathrm{O}$ como lo plantea Hernández \& Sandoval (2003) una disciplina que aborde como objeto o sujeto de estudio al humano debe considerar al lenguaje como una de las dimensiones cruciales para su entendimiento.

En la primera parte del texto, se exponen los elementos que definen desde diferentes disciplinas qué es el lenguaje; para la gramática, la lingüística, la filología, la semiología y la fonética por un lado; la antropología, la economía y la educación por otro y desde luego la psicología, psicolingüística, neurolingüística y la programación neurolingüística, (PNL). 
Incluso el lenguaje se da el lujo de tener su propia filosofía y construir la realidad, de tener un recorrido a la par con la humanidad y de lograr interrelacionarse o camuflarse casi como sinónimo con el pensamiento y la cognición; de atravesar debates infinitos como lo natural (biológico) y lo adquirido (simbólico) y de marcar distancias (para algunos), con otros seres no humanos. Al parecer son muchas las áreas interesadas en su conocimiento y desde hace mucho tiempo, es aquí, sin duda, donde yace su espíritu transdisciplinar.

En una segunda parte se desglosan las implicaciones de cada una de las particularidades del lenguaje señaladas y al final a manera de conclusión o cierre momentáneo- una interrelación entre lo anterior y la propuesta transdisciplinar ¿Se puede obtener otra perspectiva en su estudio?

\section{Desarrollo}

\section{El Crátilo. El génesis}

El diálogo platónico titulado Crátilo, es el primer registro académico que se reconoce en la historia del estudio del lenguaje. Platón (360 $A C)$, identifica la base de la lingüística al reconocer el sonido, la imagen y el signo como los elementos constitutivos del lenguaje. Su particularidad es la importancia de tres elementos constitutivos del lenguaje; la relación arbitraria del nombre a su naturaleza, rosa, sólo puede ser rosa, no piedra. De allí nace la teoría naturalista del lenguaje. Luego identifica en su debate la teoría convencional del lenguaje, donde el nombre de las cosas es dado por un grupo social de manera arbitraria. Lo ideal, finaliza Platón es la relación del significado del nombre; no es el objeto la palabra, sino la relación convencional del mundo de las ideas o significados lo fundamental. Esta herencia ha determinado el estudio del lenguaje por más de 2.500 años, su valor se expone al señalar sus particularidades y la especificidad que cada una de ellas ha dejado. Por ejemplo:

\section{Las particularidades del lenguaje}

Saussure (1945), desde la lingüística, establece las principales dicotomías en el estudio del lenguaje, al señalar la lengua- habla y desde luego el significado- significante. Aquí se conjugan esos elementos que caracterizan el lenguaje y que define Saussure como heteróclito e inclasificable. No puede estar en un solo dominio del ser; es natural, social e individual, a la vez. Fue entonces la primera mirada estructural del lenguaje e inició su estudio y a la vez, señaló, otra disciplina como la semiología al reconocer la existencia de "signos" en el seno de la vida social, diferentes a los del objeto de estudio de la lingüística.

Al mismo tiempo, con otra formación y en otro continente, Peirce (1960) planteaba desde la lógica, la filosofía y el pragmatismo, una perspectiva del lenguaje como construcción de la realidad, elaborada a través de la capacidad simbólica del ser. Para él "el hombre solo piensa en signos"y su objeto de estudio fue la semiosis (constitución de signos) que se entiende como "una acción o influencia que implica la cooperación de tres elementos, el signo, su objeto y su interpretante y esta influencia no puede, en ningún caso, reducirse a relaciones entre pares" (Niño, 2009. p 19).

Desde otra perspectiva Aitchison (1992) citado por Pinzón (2005), considera el lenguaje como un proceso físico, biológico de desarrollo ontogénico y filogenético, a partir del cual los individuos y las especies logran categorizar y conceptualizar el mundo desde su conocimiento, capacidad y habilidad para reconocerse como grupo, con necesidades, motivaciones y sentimientos comunes.

En esta definición se proyecta otros rumbos importantes de las particularidades del lenguaje y que, a la vez, confluyen en sus generalidades. Lo evidencia como elemento connatural a la evolución física y fisiológica de los seres humanos, igualmente y con un mundo de eviden- 


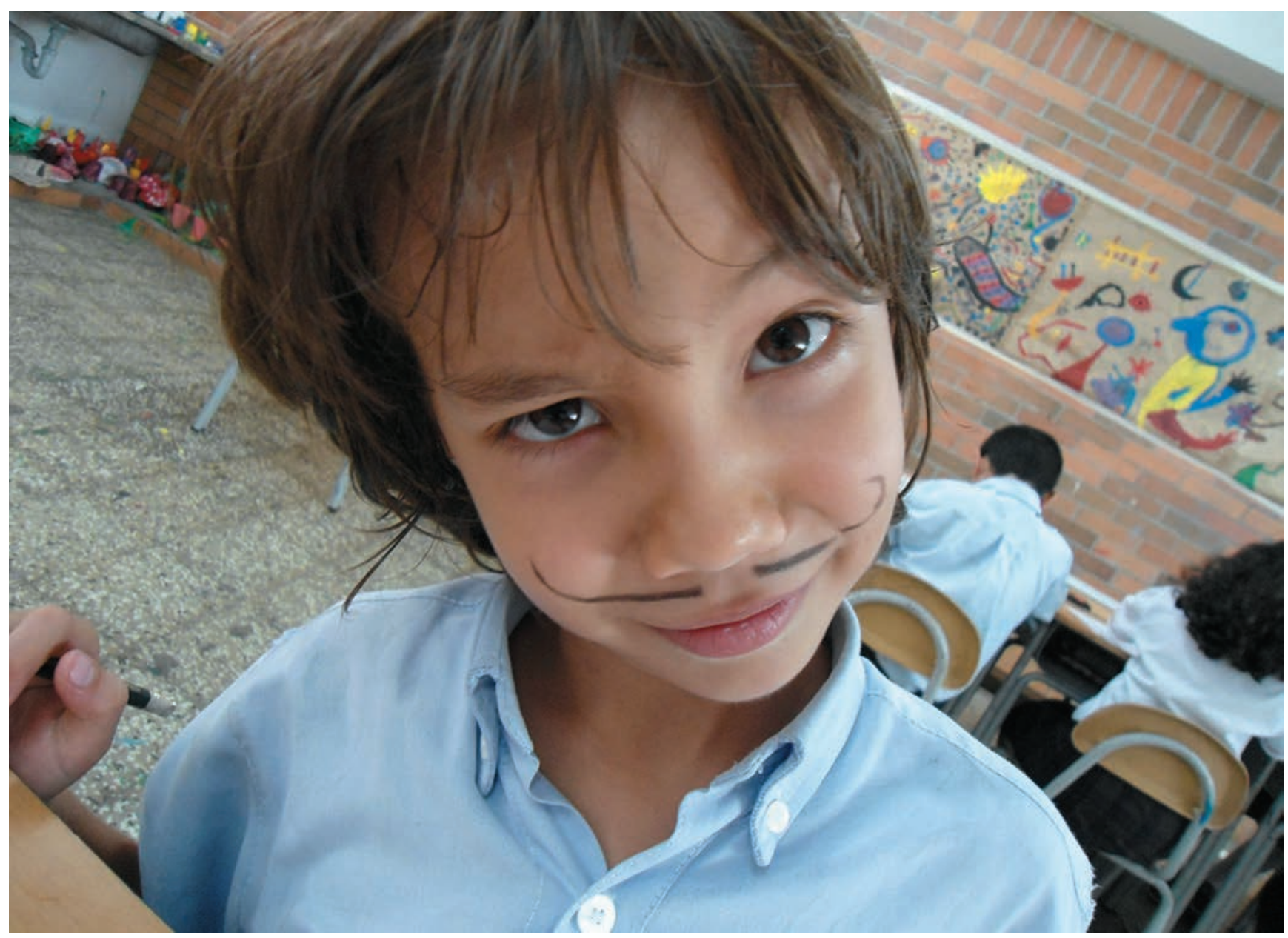

Imagen fotografía María Isabel Jasbón

cias determina la relación del lenguaje en sentido evolutivo como aspecto social y cultural. Gracias al lenguaje se intercambia conocimiento, experiencias históricas, culturales y estéticas. Rossano, (2010), ha investigado las numerables cuevas de arte rupestre e identifica que son un registro icónico claro de expresión, que permiten la construcción de teorías, de cómo se comunicaban y transmitían conocimiento los primeros humanos; y además vincula también lo icónico con el pensamiento. En sus palabras esclarece: "Allí han quedado los registros evolutivos de signos que maduraron con la especie. Son la manera actual de poder recrear la mente de los primeros hombres".

Bikerton (2005) reafirma lo expresado por Rossano al decir que la iconicidad es el camino más probable por el que llegaron nuestros ancestros al lenguaje. Para él, el lenguaje es:

"la capacidad de concatenar sistemáticamente unidades simbólicas (palabras, signos manuales) que refieren a nuestros conceptos internos de fenómenos del mundo real (entidades, acciones, estados (...) para generar proposiciones de longitud indeterminada y de entender los significados de tales proposiciones".

Su origen es determinante:

"El lenguaje sólo puede haber surgido de alguna clase de presión selectiva a la que los ancestros de la humanidad estuvieran sometidos, en este caso las situaciones de caza son su mejor ejemplo y la función pragmática inicial fue la valoración de lo bueno o malo de las conductas y los objetos". Bikerton. (2005). 
Los antropólogos dedicados al estudio de la evolución del comportamiento humano están obligados al reconocimiento social y cultural del ser, e indiscutiblemente hacer referencia al lenguaje. La necesidad de emitir y recibir información, es decir, de comunicarse se convirtió en un recurso vital para la sobrevivencia: "Ya no solo el grupo se reunió a acicalarse; fue fundamental otras expresiones de cuidado y de estrategia social como el chisme". (Dunbar, 1996. Citado por Irons, 2004).

Al llegar a este punto los estudiosos del lenguaje inmediatamente lo vinculan con pensamiento y realidad. Vigotsky citado por Baquero (1999) reconoce la comunicación como función inicial del lenguaje y en contraste con lo innato; esta comunicación es netamente social. Sin embargo esta relación está inherente a lo que llamó la ley genética de desarrollo cultural.

“Desde un plano interpsicológico se consigue lo intrapsicológico; lejos de ser solo transmisión del exterior al interior es una larga serie de procesos evolutivos que implican en un sentido literal una reconstrucción interna". (p. 53).

Estos serían los primeros pasos para la relación del pensamiento y lenguaje a través de la identificación de la conciencia. Así se alcanzó un peldaño diferencial con otras especies.

Somos capaces de llegar a una reflexión acerca del significado de nuestra existencia y desde luego, de lo que más aceleró nuestra creatividad: la muerte. Evidente en el lenguaje (icónico y simbólico) que se preserva en cuevas y tumbas. Esto identifica su esencia psicológica, como lo dijo Rossano la manera de conocer la mente de nuestros antepasados.

Un destacado lingüista Max Müller, (citado por Castro \& Toro, 2005) defendió la tesis que el lenguaje carece de cualquier antecedente animal y constituye el auténtico eslabón que separa nuestra especie de las demás.
Esta estrecha vinculación entre lo interno y lo externo y su eterno retorno, fue clave para la construcción social y de inmediato lo cultural; los mitos, los ritos y cultos han sido tema de análisis de antropólogos y demás profesionales de las ciencias sociales. El símbolo aparece como madurez del signo y las relaciones internas de grupos fueron y son los pilares de su existencia. (Rossano, 2010). Lo paliativo es necesario para explicar lo inexplicable y el mito, su oralidad; se instaura en lo cultural y cohesiona lo social.

Hasta aquí, el recorrido relaciona algunas aproximaciones que en torno al lenguaje se han realizado, dimensiona distintas disciplinas, teorías y formas de conocimiento, establece órdenes y perspectivas a través de las cuales es posible reconocer al hombre como ser biológico, individual, social y simbólico. Esta es la principal evidencia que también Pinzón (2005), describe como fundamental en el estudio del lenguaje. Pero también evidencia el protagonismo que tiene el lenguaje para la humanidad; como dice Cruz (1995): "El lenguaje nos rodea como el aire, y así como del aire depende nuestro ser biológico, del lenguaje depende nuestro ser específicamente humano" (p. 49).

Es a partir del desarrollo de las particularidades del lenguaje: lo natural o biológico, lo individual, lo social y cultural y lo simbólico que se desglosan otras relaciones:

\section{El desglose de particularidades}

"El lenguaje es una condición
de la existencia del hombre"
Octavio Paz

La lingüística es la primera en buscar apoyo. La definición de la fonética es más que evidente: Según Calderón (2001) es la ciencia encargada de estudiar los comportamientos, psicológicos, anatómicos y fisiológicos indispensables para la producción del sonido o voz humana. Sin ella indiscutiblemente no se llega a la escritura y es desde este momento, donde la sociedad 
se transforma y construye otras necesidades en torno al lenguaje; haciéndolo más complejo y multidisciplinar. La fonética es protagonista al permitir interrelacionarse con más de 28 ciencias desde la paleografía, historia, psicología, acústica, audiología, química, neurolingüística, fisiología y biología, entre otras. (p.13).

Aquí también está implícita la dicotomía de Saussure entre la lengua - habla, entre lo social- individual $y$, por qué no, entre lo hablado y lo escrito.

Hay desde luego "algo más allá" en la construcción del sentido que encadenar palabras en un orden social predeterminado ya sea de manera oral o escrita. Dos funciones del lenguaje que confluye como las caras de una misma moneda.

Para Chomsky (1985), el estudio y conocimiento del lenguaje ha sido un proceso a la par con las corrientes filosóficas, que en posiciones racionalistas o empiristas argumentan la relación mente-cerebro; el reconocimiento de una facultad lingüística y la existencia o no de una gramática generativa.

Más adelante Chomsky (1986), señala en un sentido profundo y significativo que el lenguaje es un espejo de la mente; es un producto de la inteligencia humana, creado de nuevo en cada individuo mediante operaciones que están fuera del alcance de la voluntad o la conciencia. Se puede reconocer en este avance que es un acto involuntario: "Estamos hechos para el desarrollo del lenguaje", p. 23.

Estas determinaciones iniciaron enérgicos debates entre lo natural o innato y lo adquirido, en la década de los 50 y 60. Personajes como Vygotsky, Piaget, Chomsky y Skinner tenían en el punto álgido de discusión sus aportes y desde luego el lenguaje fue protagonista de sus producciones. Chomsky fue uno de los más críticos del texto de Skinner: Conducta Verbal. Fue tan fuerte su influencia que se hicieron más famosas sus críticas que el libro de Skinner, Peña \& Robayo (2007). Indiscutiblemente la mayor debilidad del texto fue la dificultad experimental y su confusión en ver la funcionalidad en sus definiciones. Más adelante se plantearán algunas soluciones a esta versión de la conducta del lenguaje.

\section{Pensamiento y lenguaje}

Vygotsky en su enfoque socio-histórico, determina el lenguaje como un Proceso Psicológico Superior. (Baquero, 1999). Esto implica que se origina en la vida social, que es específico del hombre y que regula la acción de mismo sobre su entorno.

El lenguaje según Vygotsky citado por Baquero (1999); se caracteriza por:

"Cumplir funciones diferentes, en principio comunicativas y luego otra referida a la regulación del propio comportamiento.

Sirve como instrumento para producir efectos sobre el entorno.

Puede aportar una función reguladora del propio comportamiento.

Y de acuerdo a lo anterior, está implicado en la reorganización de la propia actividad psicológica". (p 54).

Más adelante Baquero (1999), nuevamente cita a Vygostky para identificar a la palabra; la cual establece como la unidad de análisis y de inmediato la determina como el vínculo entre el lenguaje y el pensamiento. En sus "palabras": "el pensamiento es lenguaje interior" (p 89).

\section{Cognición y lenguaje}

Son varios los autores que investigan en la cognición y el lenguaje. Uno de los más reconocidos y pionero en este trabajo es Piaget. Aunque Piaget también expone la relación pensamiento-lenguaje, en este apartado se centra sus aportes desde los procesos cognoscitivos, 
lo cual lo conecta al aprendizaje y a la inteligencia de manera constructivista. Para Piaget, antes del lenguaje existen otros procesos que el niño debe asimilar y adaptar para su desarroIlo. El desequilibrio cognitivo es constante en el desarrollo mental y sus aportes por etapas o logros en cada uno de estos procesos ubican al lenguaje como uno de los indicadores de la inteligencia y desarrollador del pensamiento. En sus palabras Piaget expresa:

"Si se examina desde más cerca los cambios de la inteligencia que se producen en el momento de la adquisición del lenguaje, se puede establecer que es uno de los responsables de estas transformaciones" (Piaget, 1991.p 112)

\section{Conducta y lenguaje}

Skinner (1981), en su obra conducta verbal es determinante en su definición del lenguaje: "el comportamiento que está mediado por una escucha, quien ha sido específicamente condicionado para mediar el comportamiento del hablante" (p10).

Se trata de un modelo operante, con tres componentes básicos: estímulo discriminativo, respuesta y reforzador. Para los conductistas el lenguaje se adquiere como cualquier otra conducta, al seguir dos principios: a) Control de las condiciones del ambiente y la crianza y b) Refuerzo de las conductas de aproximación al lenguaje adulto.

En otras palabras el ambiente es el que condiciona y refuerza la conducta verbal, los estímulos constantes en el entorno y el refuerzo social inmediato permite que se genere y mantenga la conducta verbal.

La propuesta en oposición con lo cognitivo, plantea que el lenguaje puede estudiarse experimentalmente (método científico), sin recurrir a construcciones no observables como intenciones, ideas y pensamientos; y además, su significado no es inherente a las expresiones escritas sino a las acciones de la escucha. (Ribes \& Harzem. 1990. p 26).

Aún hoy día, son muchas las críticas en torno a este determinismo ambiental, pero a favor $o$ en contra los planteamientos se han mantenido $y$, los grupos post-skinnerianos retoman y llenan los "vacíos" de ambigüedades que pudiesen ser críticas o deficiencias de la teoría conductual. La evolución de muchas de las ideas conductuales iniciales, en torno al lenguaje no fueron fáciles. Muchos de los investigadores como Kantor y Ribes, veían la necesidad de una teoría integradora al no tener una satisfacción en los planteamientos de Skinner.

El siguiente apartado es un ejemplo de ello.

\section{Actualmente la conducta del lenguaje}

La dimensión simbólica y lingüística del ser humano en la actualidad, es estudiada desde lo conductual por dos técnicas: La Nominación y la Teoría de los Marcos Relacionales TMR.

Hernández y Sandoval (2003), publicaron un análisis de estas dos técnicas e identifican la importancia del lenguaje como mediador de la relación del individuo y su ambiente natural y social. Además reconocen a la cultura como el producto simbólico adaptativo que "permite la resolución de problemas cotidianos, a través de la acumulación de soluciones y formas de entender situaciones". En su palabras: "Todo acto individual es cultural" (p74)

Además aseguran que estudiar el lenguaje es obligatorio en el estudio del comportamiento del ser humano. De acuerdo a lo anterior presentan las técnicas; definidas así:

Nominación: Propuesta por Horne y Lowe en 1996, bajo los operantes verbales de Skinner. Y se centra en la conducta del escucha y los tactos efectivos del objeto. (p.79)

T.M.R.: Se basa en un tipo de pragmatismo denominado Contextualismo funcional. Es propuesta por Hayes- Barnes- Holmes y Roche en 


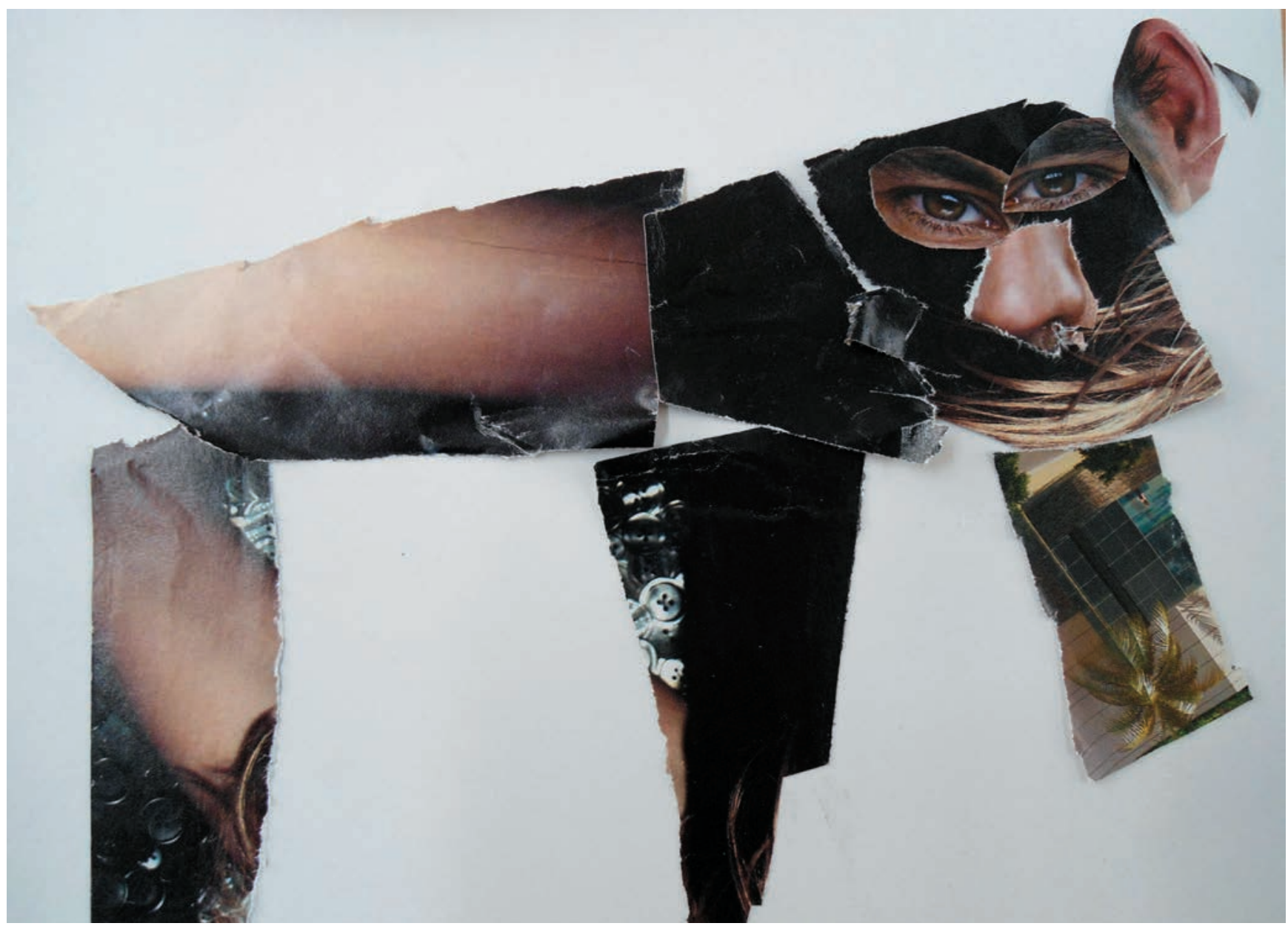

Imagen fotografía María Isabel Jasbón

los años 90. (p.80) y definen la conducta verbal como: "La acción de enmarcar eventos relacionalmente, es decir, responder a un evento en términos de otro con base a una clave contextual" (p.80).

Hay muchos más elementos en cada uno de estas técnicas que profundizan en muchas aplicaciones en diferentes contextos. Sólo es necesario para el interés de este documento su reconocimiento y su importante aporte actual sobre el lenguaje.

\section{Relaciones del lenguaje}

Psicolingüística. Barrera y Fraca (1991); consideraban a la psicolingüística como una ciencia naciente en los años 90. Es decir, que hoy en día se puede reconocer de esta forma. Ellos la definen como: "el estudio de los procesos mentales implícitos en la comprensión y emisión de mensajes articulados, en situaciones específicas de comunicación". (p.13). La psicolingüística toma elementos de la lingüística y la psicología para analizar la lengua como conocimiento y comportamiento. Se relaciona íntimamente con la psicología del lenguaje y la psicología del desarrollo o psicoevolución. Ya desde el inicio se hacía referencia a la importancia de evolución ontogénica del lenguaje. De la mano de la filogénesis del mismo, esta ciencia junto a la antropo-lingüística dan aportes a cómo nos hacemos en el lenguaje como individuos y como especie. Gehlen (1987), como antropo-biólogo asegura que la cultura es la segunda naturaleza del hombre y sólo a través del accionar del hombre en su entorno, sobrevive y se construye.

Neurolingüística. Para Fajardo \& Moya, (1999), la neurolingüística como ciencia co- 
bró importancia a partir de la Segunda Guerra Mundial. Es fácil asociar el por qué, al igual que el reconocimiento de lo patológico como misterio. Un ejemplo de esto, es el conocido caso de Phineas Gage.

El objeto de estudio de la neurolingüística es la relación existente entre la distribución cerebral, la ubicación y el desarrollo de la facultad del lenguaje. Para los autores mencionados es un amplio campo de la neuro-psicología que va más allá de la pura afasiología y que tiene como misión el estudio de las correlaciones del lenguaje con las funciones cerebrales. (p. 10).

Luria (1980) citado por Fajardo y Moya (1999); la define como la rama de la neuropsicología que estudia los mecanismos cerebrales del lenguaje y los cambios que se producen como consecuencia de lesiones focales. (p.9)

Este ámbito es protagonista en este recorrido, junto a la fonética y genética, que también profundizan en lo biológico, la neurolingüística da un encuadre natural, evolutivo y vital en el desarrollo y mantenimiento de las facultades del lenguaje. También en este espacio están los aportes de la neurofisiología del lenguaje al clarificar las estructuras anatómicas funcionales, genéticamente determinadas en el lenguaje. Todo esto no podría ser efectivo sin el estímulo del entorno. Los sistemas funcionales del lenguaje para Castaño, (2003) son:

1. Un sistema operativo o instrumental, que ocupa la región perisilviana del hemisferio dominante y que incluye el área de Broca y el área de Wernicke.

2. Un sistema semántico, que abarca grandes extensiones corticales de ambos hemisferios.

3. Un sistema intermedio organizado modularmente, que sirve de mediación entre los dos anteriores y que se ubica alrededor del sistema instrumental.
Todos estos procesos han sido identificados por "casualidad" debido al evento o presencia de alteraciones sin resolver y a partir de la investigación oportuna de las mismas.

Programación Neurolingüística. PNL. En este aspecto podemos encontrar un amplio desglose de aplicaciones, reconocimiento y nuevos lenguajes que combinan muchos de los aspectos del recorrido que hemos realizado. John Grinder y Richard Bandler en los años setenta, iniciaron con esta propuesta en los Estados Unidos, para ellos la PNL es un conjunto de poderosas técnicas diseñadas para hacer cambios permanentes en las personas a corto plazo. Ofrece la oportunidad de estudiar las estrategias que utilizan las personas de excelencia que sobresalen en diferentes áreas y utilizan técnicas efectivas que enseñan cómo alcanzar el éxito en la vida. Aseia (2012).

Su discurso de entrenamiento tiene muchos seguidores y de diferente formación profesional. En la actualidad ellos se encargan de formar por todo el mundo a través de diversas escuelas, a líderes que deseen formarse en la excelencia integral para formar a otros con el ejemplo de su éxito. Es un alocución, positiva y entusiasta que integra el poder del "lenguaje" en la percepción de la vida de cada uno de los participantes.

\section{Conclusiones}

A manera de conclusión conviene plantear la enseñanza del lenguaje como un último elemento de desglose del proceso, es el eje de integralidad del lenguaje. Si se toma como referencia los aportes de todas las ciencias nombradas hasta aquí, tanto descriptivas, teóricas, aplicadas y experimentales, su mejor campo de acción es la educación. En este espacio surgen las preguntas y las respuestas a muchos de los procesos que cada ciencia aborda. Aprender a leer y escribir es uno de los primeros descubrimientos como seres humanos que transforma la manera de ver el mundo. Esta nueva perspectiva en los niños puede convertirse en un obstáculo o un facilitador de su experiencia escolar. Sus dificultades 
en comprensión y escritura, transversalizan su relación en todas las áreas del conocimiento. Es el maestro en lo cotidiano el encargado de identificar y valorar las necesidades que presentan sus estudiantes, desde sus primeros años hasta incluso un doctorado, de las necesidades comunicativas fundamentales para su aprendizaje. En este espacio se podría generar todo un capítulo independiente del protagonismo del lenguaje en la educación.

Como se estableció desde el principio, a manera de cierre momentáneo se puede identificar la alta complejidad del lenguaje en su estudio y su cotidianidad. Es fundamental como psicólogo conocer su valor en el comportamiento, independientemente del ámbito de interés que desarrolle, desde lo cognitivo, conductual, social y neurológico, todos tocan de alguna manera al lenguaje.

Y para finalizar, solo puedo plantear más preguntas que el lenguaje genera:

¿Puede existir algo más complejo y transdisciplinar que el lenguaje? ¿Es el lenguaje el que hace complejo nuestro pensamiento? ¿Qué diría Morin de esto? Lo complejo está en lo indescifrable o inteligible es un intento de comprender y explicar la realidad con un ritmo multidimensional. ¿Cómo podría ser leído el lenguaje transdisciplinar?

Preguntas con respuestas que desde lo transdisciplinar se pueden resolver con la globalidad del ser, donde la educación ante los recursos tanto naturales como artificiales esté valorada en un lenguaje ético y estético. Donde lo cultural transcienda fronteras y límites de poder y autoritarismo; donde se instaure en cada ser su necesidad de comunicar y conocer para la libertad sin verdades definidas, sino por construir en un bien común y solidario.

\section{Referencias Bibliográficas}

Aitchison, J. (1992). Animales que intentan hablar. El mamífero articulado. Madrid: Alianza.
Aseia. (2012).¿Qué es la programación Neurolingüística? Recuperado el 26 de Noviembre de 2012 en http:// www.pnlaseia.com/

Barrera, L. \& Fraca, L. (1991). Psicolingüística y desarrollo del español. Caracas: Monte Ávila.

Baquero, R. (1999). Vigotsky y el aprendizaje escolar. Argentina: Aique

Bikerton D (2005). Entrevista documental. Recuperado el 21 de Noviembre de 2012 de http://www.desdeelexilio.com/2009/05/11/desarrollo-del-lenguaje-entrevista-a-derek-bickerton.

Calderón, A. (2001). Relaciones multidisciplinarias de la fonética. En B. Jaimes (Ed.). Lenguaje y cognición (pp. 9 -47). Bogotá: Universidad de Salamanca y el Instituto Caro y cuervo.

Castaño, (2003). Bases neurobiológicas del lenguaje. Revista de Neurología. 36 (8). 781-785.

Castro, L. \& Toro M. (2005). La evolución del Lenguaje. Ludus vitalis XIII, (24), 203 -210 Recuperado el 23 Noviembre, 2012, de http://www.ludusvitalis.org/ textos/24/24_castro-toro.pdf.

Chomsky, N (1985). El conocimiento del lenguaje. Su naturaleza, origen y uso. Madrid: Alianza.

Chomsky, (1986). Reflexiones acerca del lenguaje. México: Trillas.

Cruz, D. (1995). El misterio del lenguaje. Bogotá: Planeta.

Echeverría; R. (1994). La ontología del lenguaje. Chile: Dolmen Ediciones

Fajardo L. \& Moya C. (1999). Fundamentos neuropsicológicos del lenguaje. Bogotá: Instituto Caro y Cuervo.

Gehlen. A. (1987). El hombre su naturaleza y lugar en el mundo. En A. Gehlen, El Hombre. (Pp. 35-45). Salamanca: Sígueme.

Hayes, S. C., Barnes-Holmes, D. \& Roche, B. (2001). Relational frame theory: A Post Skinneriana account of human languaje. New York: Kluwer Academic

Hernández, A. \& Sandoval, M. (2003). La actividad simbólica humana: Una revisión de las técnicas contemporáneas en el análisis del comportamiento verbal. Acta Colombiana de Psicología, 10, 73-87.

Hernández, F. (s. f.) Las relaciones entre pensamiento y lenguaje según Piaget, Vygotsky, Luria y Bruner. Recuperado el 27 Nov. 2012 en http://digitum.um. es/ 
xmlui/bitstream/10201/21940/1/05\%20Las\%20relaciones\%20entre\%20pensamiento\%20segun\%20Piaget\%20Vygotsky.pdf

Irons, W. (2004). How has evolution shaped human behavior? Richard Alexander's contribution to an important question. Evolution and human behavior $26 \mathrm{pp}$. $1-9$.

Niño, V. (2009). Fundamentos de semiótica y lingüística. Bogotá. D.C. : Ecoe

Niño, V.(2002). Semiótica y Lingüística. Aplicados al Español. Bogotá. Ecoe.

Peirce; Ch. (1960) Collected papers of Charles Sanders Peirce Recuperado el 26 de enero de $2012 \mathrm{en:} \mathrm{http://www.}$ centro-de-semiotica.com.ar/Peircelnterpr. html.

Peña, T. \& Robayo, B. (2007). Conducta verbal de B. F. Skinner: 1957-2007. Revista Latinoamericana de Psicología. 39, 3, 653 -661.

Pérez, M. Barrios M. \& Zuluaga Z.(2010). ¿Cómo se enseña lenguaje en Colombia? En Palabra Maestra. 10 (24), 5-8.

Piaget, J. (1991). Seis estudios de psicología. Barcelona: Labor.
Pinzón, S. (2005). Lenguaje, lengua, habla, idioma y dialecto. Revista la Tadeo. Lenguas del mundo. 71, 9-21.

Platón (360 AC). Crátilo, en Diálogos. Obra completa en 9 volúmenes. Volumen II: Gorgias. Menéxeno. Eutidemo. Menón. Crátilo. (Pp. 358-455). Madrid: Gredos.

Reyes, R. (2006). Lenguaje e investigación. Recuperado el 20 de noviembre de 2012 en: http://educacionbabel. blogspot.com/2008/02/un-plateamiento-transdisciplinar.html

Ribes, E. \& Harzem, P. (1990). Lenguaje y conducta. México D.F.:Trillas.

Rossano, M (2010). Making Friends, Making tools and Making symbols. Current Anthropology 51- 1 89-97.

Saussure de, F. (1945) Curso de Lingüística General. Buenos Aires: Losada.

Skinner B. F. (1981). Conducta Verbal. Bogotá: Trillas

Vargas-Mendoza, J. E. (2006) Bases de la teoría de los marcos relacionales: Apuntes para un seminario. México: Asociación Oaxaqueña de Psicología A.C.

\section{Cómo citar este artículo:}

Garnica, M. L. (2013). El lenguaje complejo diferente al complejo lenguaje. Espiral, Revista de Docencia e Investigación. 2 (2), 59-68 